\title{
Application of the Microhole and Strip Plate Detector for Neutron Detection
}

\author{
J. F. C. A. Veloso, F. Amaro, J. M. F. dos Santos, J. A. Mir, G. E. Derbyshire, R. Stephenson, N. J. Rhodes, and \\ E. M. Schooneveld
}

\begin{abstract}
We introduce the microhole and strip plate (MHSP) detector as a micropattern detector for the detection of thermal and epithermal neutrons. Detection sensitivity is obtained by filling these detectors with ${ }^{3} \mathrm{He}$ at high pressures. We propose the use of argon-xenon penning mixtures as the stopping gas as opposed to the usual carbon based stopping gases. These argon-xenon mixtures provide suitable gas gains for the high pressure/high resolution neutron detector applications. With these mixtures it is possible to obtain a sealed detector with only rare-gas filling which is simple to purify and not subject to ageing.

An MHSP gas detector filled with a 3-bar argon/50-mbar xenon/6-bar helium mixture can achieve gains above $2 \times 10^{3}$. This mixture allows neutron detection efficiencies of about $70 \%$ at $1 \AA$ for a $2.5-\mathrm{cm}$ thick absorption region and intrinsic position resolution (full-width at half-maximum) of about $1.8 \mathrm{~mm}$. The sensitivity to $\gamma$-rays of the present mixture will be the same when compared to that of 2.6-bar $\mathrm{CF}_{4}$
\end{abstract}

\section{INTRODUCTION}

A NEW generation of neutron gas-detectors to meet the requirements of new neutron spallation sources [1]-[8] is under development. Neutron applications include the use in 2-D position sensitive beam monitors, reflectometers, and single crystal diffractometers. Typical requirements for the future detectors include:

i) neutron detection efficiency of $\sim 70 \%$ at $1 \AA$;

ii) position resolution of about $1 \mathrm{~mm} \times 1 \mathrm{~mm}$;

iii) time resolution in the $\mu$ s range;

iv) large area, larger than $200 \mathrm{~mm} \times 200 \mathrm{~mm}$ sensitive area;

v) count rates up to $1 \mathrm{MHz} / \mathrm{mm}^{2}$;

vi) low $\gamma$-sensitivity.

With the introduction of the microstrip gas chamber (MSGC) by Oed [9], applications to neutron detection have been carried out with success [3], [5]. Additionally, other micropattern detectors such as microgap chambers [10], pin-pixel detectors [2], and detectors based on the gas electron multiplier (GEM) [4], [6] have been investigated.

Manuscript received November 13, 2003; revised March 12, 2004 and May 26, 2004. This work was supported by Project POCTI/FNU/50360/2002. The work of J. F. C. A. Veloso was supported by Fundação para a Ciência e Tecnologia, Portugal.

J. F. C. A. Veloso is with the Physics Department, University of Aveiro, P-3810-193 Aveiro, Portugal, and with the Physics Department, University of Coimbra, P-3004-516 Coimbra, Portugal (e-mail: jveloso@gian.fis.uc.pt).

F. Amaro and J. M. F. dos Santos are with the Physics Department, University of Coimbra, P-3004-516 Coimbra, Portugal.

J. A. Mir, G. E. Derbyshire, and R. Stephenson are with the Instrumentation Department, CCLRC Rutherford Appleton Laboratory, OX11 0QX Oxon, U.K.

N. J. Rhodes and E. M. Schooneveld are with ISIS, CCLRC Rutherford Appleton Laboratory, OX11 0QX Oxon, U.K.

Digital Object Identifier 10.1109/TNS.2004.835620
Gas detectors utilising ${ }^{3} \mathrm{He}$ under high pressures present an excellent option for neutron detection, since other neutron-counting gases based on high-pressure ${ }^{10} \mathrm{BF}_{3}$ present the drawback of electron attachment to the molecule, which is strongly electronegative.

The thermal-neutron capture reaction by ${ }^{3} \mathrm{He}$ can be described as follows:

$$
{ }^{3} \mathrm{He}+{ }^{1} \mathrm{n} \rightarrow{ }^{3} \mathrm{~T}(192-\mathrm{keV})+{ }^{1} \mathrm{p}(572-\mathrm{keV}) .
$$

The detection efficiency depends on the gas pressure and the thickness of the absorption region. While ${ }^{3} \mathrm{He}$ under high-pressure can provide suitable neutron detection efficiency for 1- to 2-cm thick absorption regions, other gases are needed to achieve the required position resolution. For instance, for 1.8 -to $1-\AA$ neutrons, 6 to 8 bar of ${ }^{3} \mathrm{He}$ presents neutron detection efficiencies of about $70 \%$ for $1-$ to $2-\mathrm{cm}$ thick absorption regions. The proton range is rather long for this gas, about $8 \mathrm{~mm}$. Thus, one needs to add to the detector volume an additional gas with heavy atoms or molecules, the so-called stopping gas, to reduce the proton range down to the required values.

The point of interaction of the neutron is determined by the centroid of the charge density of the ionization produced along the proton- and triton-tracks. However, this centroid does not coincide with the neutron interaction-position due to the fact that the proton track is three times longer than the triton track, with the highest ionization density at the end of the tracks. The centroid distribution around that interaction-point results in an intrinsic position resolution, full-width at half-maximum (FWHM), which is approximately $70 \%$ of the proton range [4], [8].

In general, carbon-based gases are used for efficient protonstopping, presenting the additional advantage of a high insensitivity to $\gamma$-rays [1], [7]. $\mathrm{CF}_{4}$ is the most commonly used gas, but the best position resolutions have been achieved with propane [7]. ${ }^{1}$

In this paper we investigate the possibility of applying the microhole and strip plate (MHSP) to neutron detection. Furthermore, we propose the use of Ar-Xe based mixtures as a stopping gas, as a replacement for the carbon-based ones. This solution would provide a detector with only rare-gas filling that is easily and efficiently purified by nonevaporable getters, resulting in a stable, long-lived, sealed detector.

${ }^{1}$ http://www.inst.bnl.gov./GasDetectorLab/NNNeutronDetectors/Brochure/ Brochre.html. 


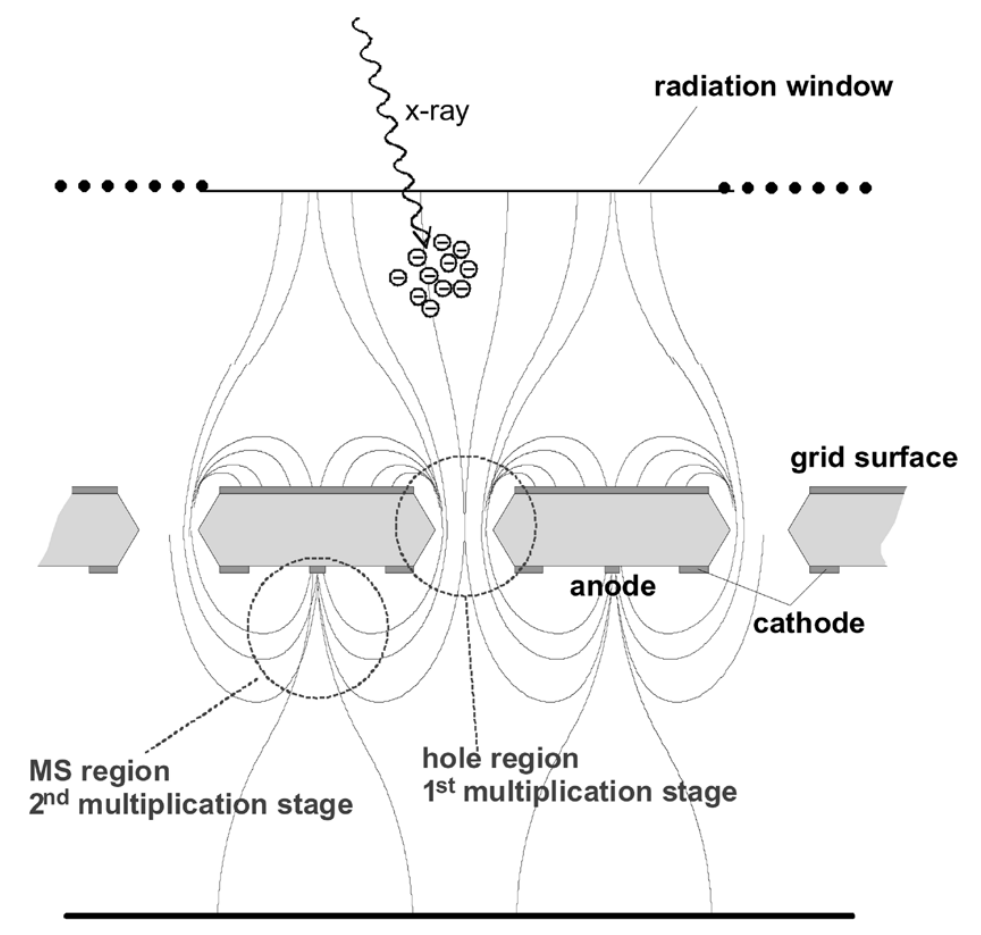

Fig. 1. Schematic diagram of the MHSP operation principle.

\section{DETECTOR DESCRIPTION}

\section{A. The MHSP Detector}

The MHSP [11], [12] is a new gaseous multiplier conceived as a combination of the features of the MSGC and the GEM in a single, double sided element. It integrates two successive independent stages of charge amplification on a single device, in a similar way as the GEM+MSGC multiplier [13].

Like the GEM, the MHSP is fabricated using printed circuit board (PCB) technology. Both sides of a $50 \mu \mathrm{m}$ Kapton film are metallized with 5- $\mu \mathrm{m}$ thick copper-layers. On the top side, a GEM-like pattern of holes is etched-through to the bottom side, where a standard microstrip pattern is etched with the holes centered in the cathode strips, and the anodes running between them.

A schematic diagram of the MHSP operation is presented in Fig. 1. Electrons produced in the drift region, above the topside, drift along field lines toward the holes and undergo charge multiplication inside them. The avalanche electrons emerge on the other side, and are directed toward the anode strips, initiating a second charge avalanche amplification.

Compared to GEMs, the presence of two electron-avalanche amplification stages allows the use of anode-to-cathode voltages that are well below breakdown thresholds, for a given overall gain. Additionally, the small distances between the three electrodes result in a fast collection of most of the avalanche ions, providing quasi-symmetric signals, as fast as 6-ns rise-time and 12-ns pulse width [12]. In principle, MHSPs can handle the high rates required for future neutron detectors, and can be made less sensitive to discharges.

Cost-effective, large-area detectors can be built, for they are produced with standard PCB technology. Together with its 2-D capability, these are compelling reasons to investigate the application of MHSPs to neutron detection. One coordinate is provided by the anode strips, while the second coordinate can be obtained by structuring the top electrode of the MHSP with orthogonal strips (see [11, Fig. 2]). An alternative approach is to obtain the second coordinate, or both coordinates, from the positive ions collected at a patterned cathode-plane placed a few hundred micrometers below the MHSP, taking advantage of the large number of positive ions, produced in the final amplification stage, that can be diverted toward it.

\section{B. The Stopping Gas}

A drawback to the use of pressurised gaseous detectors and, in particular, micropattern detectors such as MSGCs, GEMs, and MHSPs, relates to the fact that detector gain drops with increasing gas pressure [4], [6], [14], [15]. For a single GEM detector, $\mathrm{CF}_{4}$ filling-pressures above 2 bar deliver gains below ten, too low to be of any use in neutron detection [4]. For other, more complex carbon-based molecules, such as propane and $\mathrm{C}_{3} \mathrm{~F}_{8}$, the situation is even worse, and it is not possible to operate the GEM with suitable gains at the required filling pressure [4]. A cascade of GEMs [6] will result in higher gains. However, a compromise has to be established between the gain achieved in each GEM and the transfer field between the GEMs. This field must be high enough to satisfy the full transparency of the first GEM, and low enough to satisfy that of the second GEM [6]. Additionally, interactions occurring in the detector volume between the GEMs will contribute to the background.

The use of another type of stopping gas that could deliver higher charge avalanche gains, in substitution of carbon-based gases, could present an alternative solution to overcome this problem. Investigations along this line are under way and may 


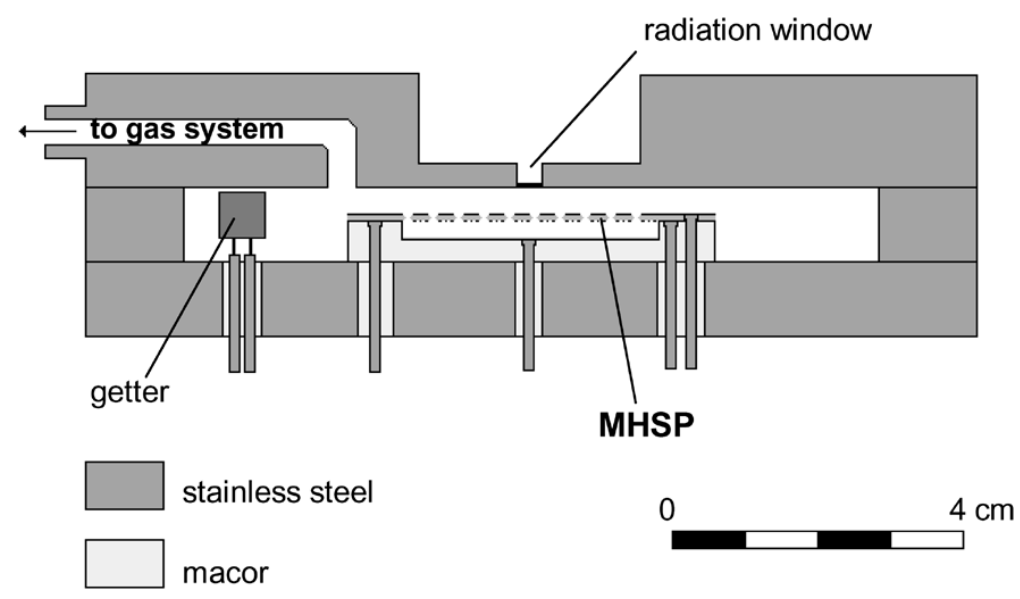

Fig. 2. Schematic of the MHSP gas detector used in this work.

present significant progress to the use of microstructures in neutron detection.

Penning mixtures allow higher gains to be achieved by promoting ionization of one of the components, which appears in a few percent content, as a result of the energy transfer from the excited molecules of the other component. Xenon-based penning mixtures have been investigated [4], [6] and it has been demonstrated that a single GEM can be operated in Xe-2.5\% trimethylamine (TMA) with suitable gains, at high pressures. Gains of about 100 have been obtained for a single GEM operating at 4-bar xenon and 200-mbar TMA, the pressure required for a 1-mm position resolution [4]. However, a drawback of using xenon as a stopping gas results from its high sensitivity to background gamma-radiation [4].

The use of argon as a stopping gas may present an alternative solution, since its sensitivity to gamma radiation is much less than that of xenon. However, a compromise has to be made between the achieved position resolution and the argon filling pressure. Gains of about $10^{5}$ have been obtained for a double-GEM multiplier operating at 1 bar of Ar-10\% DME, dropping to gains of about 100 at 7-bar fillings [6].

The use of penning mixtures, such as argon-xenon, is known to deliver high gains with low applied voltages. At 1-bar filling pressure the best operation conditions, i.e., highest gains with the lowest biasing voltages, are obtained for xenon concentrations around 5\% [16], [17], and gains above $10^{4}$ have been obtained [18] with a single MHSP. Additionally, argon-xenon mixtures present the advantage of being free of complex molecules, providing a detector with only rare-gas filling that is easily and efficiently purified by small nonevaporable getters. Such detectors can be sealed, very long-lasting, and not subject to aging, and, therefore, used in very high radiation environments.

\section{EXPERIMENTAL SETUP}

A small detector prototype was built to accommodate the MHSP microstructure inside a small gas volume (Fig. 2). The detector body was made of stainless steel. Macor pieces, simply glued with low vapor-pressure epoxy to the stainless-steel body, were used for insulating the feedthroughs of the detector biasing and of the purifier power-supply. The MHSP foil was mounted on a Macor frame to keep it stretched and to provide the different electrical contacts to the MHSP, as well as to the backplane electrode. The detector radiation window is made of $25-\mu \mathrm{m}$ thick Kapton foil, aluminized on the inner surface to ensure electrical conductivity. It was made small, $2 \mathrm{~mm}$ in diameter, to withstand the high-pressure gradients, planned for this experiment, but a multiple window pattern, regularly distributed over the whole MHSP active area, can be implemented.

The MHSP has an active area of $28 \times 28 \mathrm{~mm}^{2}$ and was placed at a distance of $5 \mathrm{~mm}$ from the radiation window and $3 \mathrm{~mm}$ from the backplane electrode, a chromium thin-film, vacuum-evaporated on the Macor surface. The MHSP hole dimension is about 40 and $60 \mu \mathrm{m}$ on the Kapton and the copper layer, respectively. The anode and cathode strip widths are 35 and $100 \mu \mathrm{m}$, respectively, with a pitch of $200 \mu \mathrm{m}$.

The detector was connected through a valve to the vacuum/gas-filling system and was sealed during the experiments. Two small nonevaporable getters, with built-in heating resistors, were placed inside the detector volume to maintain gas purity. At the maximum filling-pressure that the detector was operated ( 9 bar), the gas-leak rate out of the detector was below 0.02 bar/hour, allowing stable operating conditions during each run.

Detector signals were fed through a Canberra 2006-preamplifier (with a sensitivity of $1.5 \mathrm{~V} / \mathrm{pC}$ ) and a Tennelec TC243 linear-amplifier (4- $\mu$ s shaping time, 8 - $\mu$ s peaking time) to a Tennelec 1024-multichannel analyzer. The electronic chain sensitivity was calibrated for absolute gain determination, using a calibrated capacitor directly connected to the preamplifier input and to a precision pulse-generator.

For these studies, we used 22.1-keV X-rays, obtained from a ${ }^{109} \mathrm{Cd}$ source, and the gain was determined from the peak-position of the pulse-height distribution. The use of the $22.1-\mathrm{keV}$ X-rays, instead of 5.9-keV, allowed a clear separation of the peak distribution from the electronic noise tail, at reduced detector gains.

\section{EXPERIMENTAL RESULTS AND DISCUSSION}

We started to study the MHSP gains obtained for the Ar-5\%Xe mixture at different filling-pressures, up to a maximum of 3 bar. 


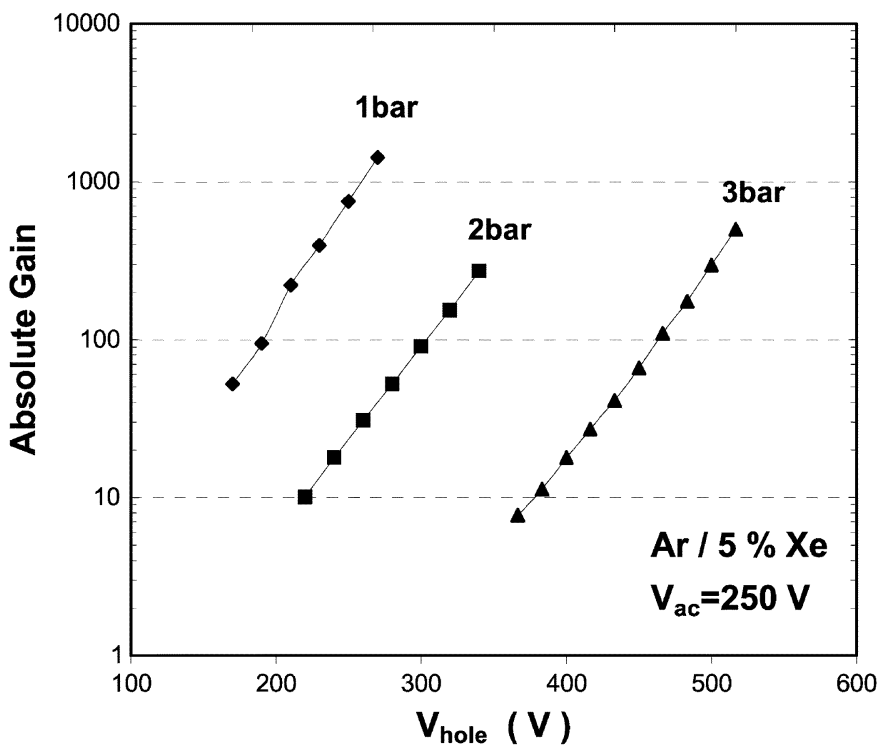

Fig. 3. Detector gain as function of $\mathrm{V}_{\text {hole }}$ for different $\mathrm{Ar}-5 \% \mathrm{Xe}$ gas filling-pressures and for constant anode-to-cathode strips voltage $\left(V_{\mathrm{ac}}=250\right.$ $\mathrm{V})$, and electric field intensities of 100 and $-70 \mathrm{Vcm}^{-1}$ in the absorption and in the induction region, respectively.

In Fig. 3 we depict the detector gain as function of the voltage difference across the holes, $\mathrm{V}_{\text {hole }}$, for Ar-5\% Xe filling pressures of 1,2, and 3 bar. The anode-to-cathode voltage difference, $\mathrm{V}_{\mathrm{ac}}$, was kept at $250 \mathrm{~V}$. The electric field intensity in the drift region, $E_{d}$, as well as that of the induction region, $E_{\text {ind }}$, were kept constant at 100 and $-70 \mathrm{Vcm}^{-1}$, respectively. To avoid the destruction of the MHSP, both $\mathrm{V}_{\text {hole }}$ and $\mathrm{V}_{\mathrm{ac}}$ were maintained below the onset of discharges, except for the 3-bar case, where the maximum gain was reached for voltages just below this onset.

The curves exhibit the characteristic exponential behavior of proportional charge avalanche processes. As shown in Fig. 3, the maximum gains achieved for the described conditions decrease with gas pressure. Nevertheless, the obtained gains are significantly high in all cases, decreasing from $1.5 \times 10^{3}$ at 1 bar to $6 \times 10^{2}$ at 3 bar.

Since the electron-impact cross-section is higher for xenon than for argon, the xenon atoms play a more important role in the charge avalanche processes. Thus, the increase of argon content has a small effect on the mean free path for xenon ionization, and constant xenon partial pressure content could, in principle, present roughly the same maximum gains.

In Fig. 4 we present the detector gain as a function of $\mathrm{V}_{\text {hole, }}$, for 1-, 2-, and 3-bar argon partial pressures, while maintaining the xenon partial pressure at 50 mbar (5\% of 1 bar), and for the same operation conditions as for Fig. 3. As seen, the maximum gain that can be achieved is about the same for the three cases, reaching a value of about $2.5 \times 10^{3}$ for the 3 -bar $\operatorname{argon} / 50$ mbar xenon mixture, one order of magnitude higher than that obtained with 3 bar Ar-5\%Xe mixture for the same voltages. A gas mixture of 3-bar argon and 50-mbar xenon has, then, been considered as the stopping gas. Nevertheless, future work will include the study of the xenon concentration that delivers high gains with lower voltages, for argon-xenon mixtures at the filling-pressures considered for our neutron detector.

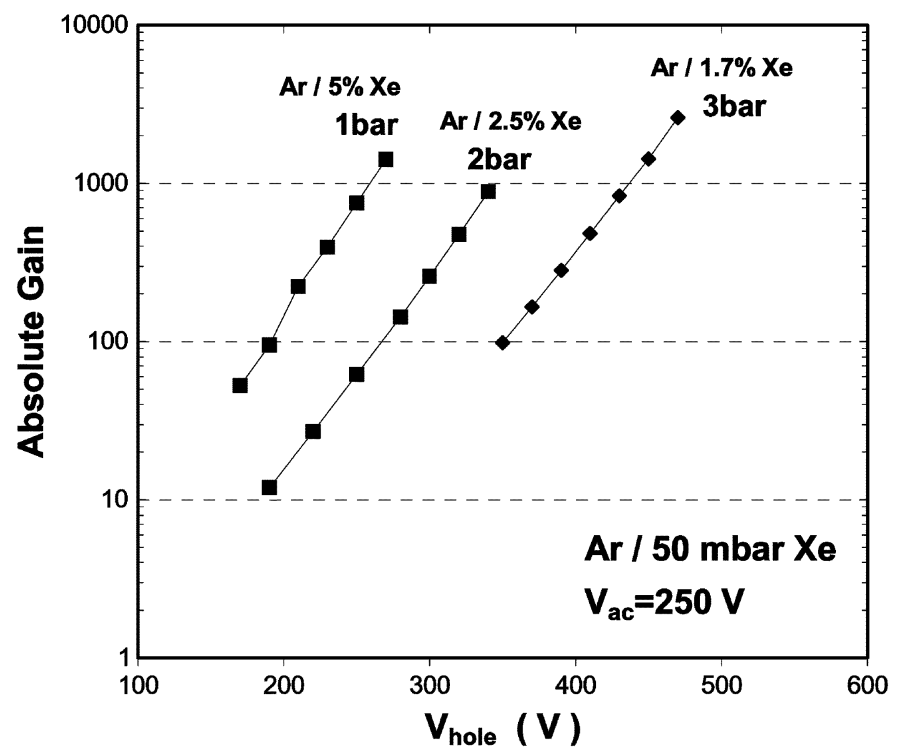

Fig. 4. Detector gain as function of $\mathrm{V}_{\text {hole }}$ for a xenon partial pressure of 50 mbar and for different argon filling-pressures. The anode-to-cathode strips voltage, the electric field intensity in the absorption region, and that and in the induction region were kept constant, at $250 \mathrm{~V}, 100 \mathrm{Vcm}^{-1}$, and $-70 \mathrm{Vcm}^{-1}$, respectively.

TABLE I

572-keV Proton Range IN PURE ARgon AND IN DifFERENT ARGON-HELIUM MIXTURES AT NPT

\begin{tabular}{|c|c|}
\hline Gas & $\begin{array}{c}\text { Proton range } \\
(\mathrm{mm})\end{array}$ \\
\hline 3-bar Ar & 3.77 \\
\hline 6.5-bar Ar & 1.74 \\
\hline 3-bar Ar / 50-mbar Xe /6-bar He & 2.59 \\
\hline 6.5-bar Ar / 50-mbar Xe /6-bar He & 1.44 \\
\hline 6-bar Ar / 50-mbar Xe /8-bar He & 1.46 \\
\hline
\end{tabular}

Finally, we investigated the effect of the addition of 6 bar of helium to the above mixture. Fig. 5 presents the detector gain as function of $\mathrm{V}_{\text {hole }}$ for different helium partial pressures added to the 3 bar of Ar/50-mbarXe mixture.

As in [4], the addition of helium hardly affects the maximum gain achieved with the argon-xenon mixture. The electron-impact cross-section for helium is much smaller than for argon and/or xenon, and the first excitation level of the helium atom, $19.8 \mathrm{eV}$, is well above the argon and/or xenon ionization levels (15.8 and $12.1 \mathrm{eV}$, respectively). Therefore, electrons will only interact elastically with the helium atoms, losing only a small amount of energy, without further affecting the avalanche gain. As shown in Fig. 5, only a voltage increase of $60 \mathrm{~V}$ was necessary to achieve the same gains as the helium pressure increased from zero to 6 bar.

As referred in Section II-B, a compromise has to be made between the argon partial pressure and the position resolution that can be achieved in the detector. Table I shows the proton stopping power range for different argon-pressures and for argonxenon helium mixtures with two different helium partial pressures, 6 and 8 bar, as calculated with the SRIM software. ${ }^{2}$ For

\footnotetext{
${ }^{2} \mathrm{http}: / /$ www.research.ibm.com/ionbeams/SRIM/SRIMLEGL.HTM.
} 


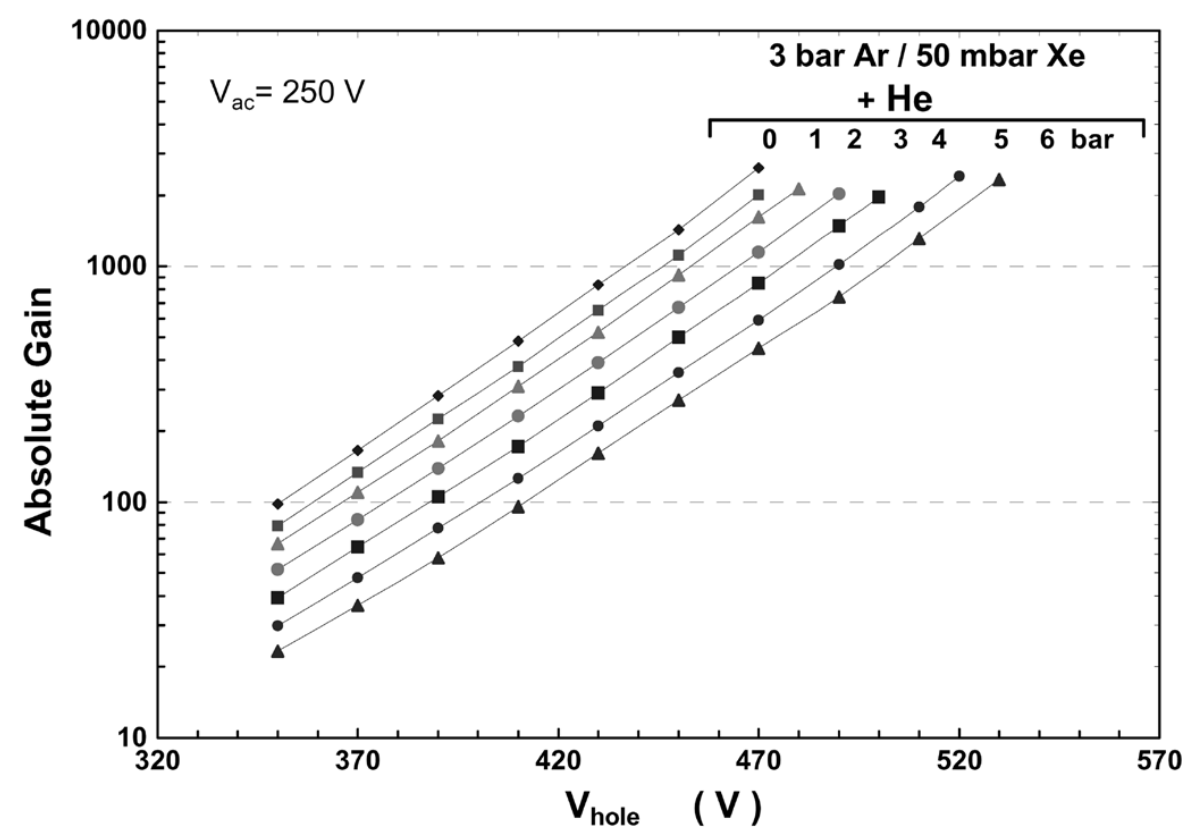

Fig. 5. Detector gain as function of $\mathrm{V}_{\text {hole }}$ for different helium partial pressures added to a 3-bar Ar/50-mbar Xe mixture, keeping the anode-to-cathode strips voltage at $250 \mathrm{~V}$, and electric field intensities of 100 and $-70 \mathrm{Vcm}^{-1}$ in the absorption and in the induction region, respectively.

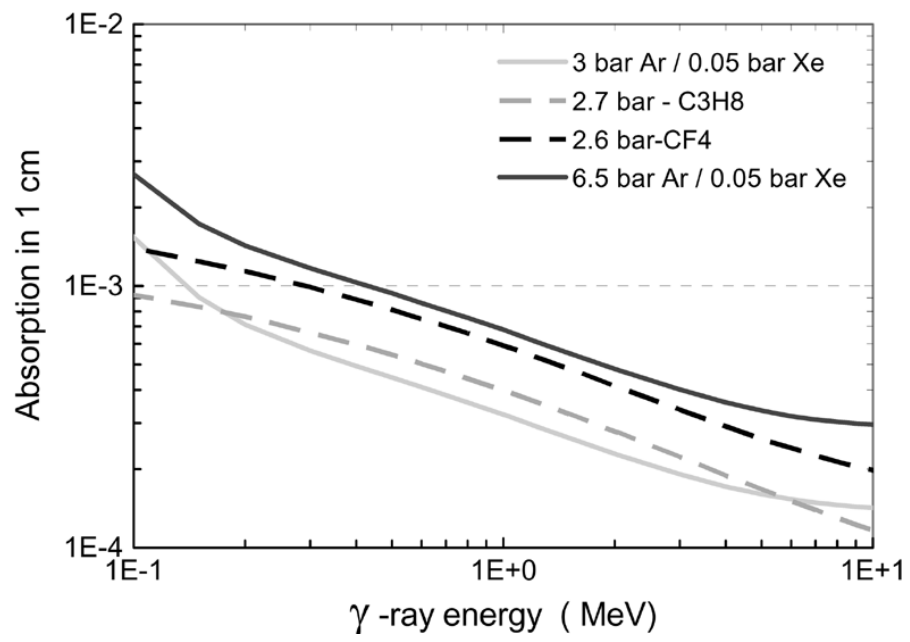

Fig. 6. Calculated photon absorption in $1 \mathrm{~cm}$ of 3-and 6.5-bar argon/50-mbar xenon mixture, 2.7-bar propane, and 2.6-bar $\mathrm{CF}_{4}$.

the present mixture, the proton range is about $2.6 \mathrm{~mm}$, leading to an intrinsic spatial resolution of about $1.8 \mathrm{~mm}$ for neutron detection ( $70 \%$ of the proton range). This can be reduced to 1.0 $\mathrm{mm}$ for 6.5-bar argon/6-bar helium mixture. Future work will include the study of the MHSP-detector gain with argon-fillings up to 6.5 bar pressure.

The $\gamma$-sensitivity of argon-xenon gas mixture is another characteristic that has to be taken into account when it is used as a neutron stopping-gas. Fig. 6 depicts the calculated photon absorption for 3- and 6.5-bar argon/50-mbar xenon mixture, 2.7-bar propane, and 2.6-bar $\mathrm{CF}_{4}$, using XCOM software ${ }^{3}$ considering the total cross-section. As shown, for the energy region of interest the $\gamma$-sensitivity for 6 bar of argon mixture is similar to that of $\mathrm{CF}_{4}$ and only $50 \%$ higher than that of propane, while 3-bar argon mixture presents slightly lower gamma sensitivity than that of propane. On the other hand, it is noted that gamma

\footnotetext{
${ }^{3}$ http://physics.nist.gov/PhysRefData/Xcom/Text/XCOM.html.
}

absorptions in the detector window may produce a contribution to background that can be significant, or even dominant, when compared to that resulting from the gamma interactions in the gas. Nevertheless, $\gamma$-rejection by pulse-shape discrimination presents a mean to further reduce $\gamma$-background events.

\section{CONCLUSION}

The feasibility of producing a ${ }^{3} \mathrm{He}$ neutron-detector based on the MHSP, and argon-xenon penning-mixture as a stopping gas, has been demonstrated. Such a mixture will render a sealed detector with only rare-gas filling, simple to purify and not subject to aging.

Gains above $2 \times 10^{3}$ were obtained for a 3-bar argon/50-mbar xenon/6-bar helium mixture. This mixture allows neutron detection efficiencies of about $70 \%$ at $1 \AA$ for a 2.5 -cm thick absorption region, intrinsic position resolution (FWHM) of about $1.8 \mathrm{~mm}$ and charge gains above $2 \times 10^{3}$. 
Position resolutions of $1 \mathrm{~mm}$ may be achieved with argon partial pressures of 6.5 bar. Future work has to be done to investigate the achieved gains and the best xenon concentration for such argon partial pressures. It is expected that the $\gamma$-sensitivity of this mixture will be similar to that of 2.6-bar $\mathrm{CF}_{4}$.

\section{ACKNOWLEDGMENT}

The authors acknowledge A. Breskin and R. Chechik (Weizmann Institute of Science, Israel) and B. Guerard (Institut LaueLangevin, France) for helpful discussions.

\section{REFERENCES}

[1] C. E. W. van Eijk, "Neutron PSD's for the next generation of spallation neutron sources," Nucl. Instrum. Meth., vol. A477, pp. 383-390, 2002.

[2] J. E. Bateman, J. F. Connolly, G. E. Derbyshire, D. M. Duxbury, A. S. Marsh, N. J. Rhodes, E. M. Schooneveld, J. E. Simmons, and R. Stenphenson, "The pin pixel detector-neutron imaging," Nucl. Instrum. Meth., vol. A485, pp. 596-605, 2002.

[3] J. E. Bateman, N. J. Rhodes, and R. Stenphenson, "A high-resolution, imaging neutron beam monitor using gas microstrip technology," Nucl. Instrum. Meth., vol. A477, pp. 165-171, 2002.

[4] R. Kreuger, C. W. E. Van Eijk, F. A. F. Fraga, M. M. Fraga, S. T. G. Fetal, R. W. Hollander, L. M. S. Margato, and T. L. Van Vuure, "Performance of high pressure Xe/TMA in GEM's for neutron and X-ray detection," in Conf. Rec. 2001 IEEE Nucl. Sci. Symp., San Diego, CA, Nov. 4-10, 2001.

[5] J. F. Clergeau, P. Convert, D. Feltin, H. E. Fisher, B. Guérard, T. Hansen, G. Manzin, A. Oed, and P. Palleau, "Operation of sealed microstrip gas chambers at ILL," IEEE Trans. Nucl. Sci., vol. 48, pp. 1075-1080, 2001.

[6] T. L. van Vuure, C. E. W. van Eijk, F. A. F. Fraga, R. W. Hollander, and L. M. S. Margato, "High-pressure GEM operation aiming at thermal neutron detection," IEEE Trans. Nucl. Sci., vol. 48, pp. 1092-1094, 2001.

[7] Proc. Eur. Workshop Thermal Neutron Detectors for the European Spallation Source (NEUDESS98), Oct. 27-28, 1998, ISO@ iri.tudelft.nl.
[8] T. Wilpert, "Overview of neutron detectors," in Proc. Eur. Workshop Thermal Neutron Detectors for the European Spallation Source (NEUDESS98), Oct. 27-28, 1998, ISO@ iri.tudelft.nl.

[9] A. Oed, "A position sensitive detector with microstrip anode for electron multiplication with gases," Nucl. Instrum. Meth., vol. A263, pp. 351-359, 1988.

[10] F. D. van den Berg, V. R. Bom, C. E. W. van Eijk, R. W. Hollander, P. M. Sarro, H. Schellevis, M. W. Johnson, and N. J. Rhodes, "A neutron detector based on the microgap counter," in Proc. Eur. Workshop Thermal Neutron Detectors for the European Spallation Source (NEUDESS98), Oct. 27-28, 1998, ISO@ iri.tudelft.nl.

[11] J. F. C. A. Veloso, J. M. F. dos Santos, and C. A. N. Conde, "A proposed new microstructure for gás radiation detectors: The microhole and strip plate," Rev. Sci. Instrum., vol. 71, pp. 2371-2376, 2000.

[12] J. M. Maia, J. F. C. A. Veloso, J. M. F. dos Santos, A. Breskin, R. Chechik, and D. Mörmann, "Advances in the micro-hole \& strip plate gaseous detector," Nucl. Instrum. Meth., vol. A 504, pp. 364-368, 2003.

[13] R. Bouclier, W. Dominik, M. Hoch, J. C. Labbe, G. Million, L. Ropelewski, F. Sauli, A. Sharma, and G. Manzini, "New observations with the gas electron multiplier (GEM)," Nucl. Instrum. Meth., vol. A 396, pp. 50-66, 1997.

[14] A. Bondar, A. Buzulutskov, F. Sauli, and L. Schekhtmann, "High- and low-pressure operation of the gas electron multiplier," Nucl. Instrum. Meth., vol. A 419, pp. 418-422, 1998.

[15] A. Bondar, A. Buzulutskov, and L. Schekhtmann, "High pressure operation of the triple-GEM detector in pure $\mathrm{Ne}, \mathrm{Ar}$, and Xe," Nucl. Instrum. Meth., vol. A 481, pp. 200-203, 2002.

[16] A. Buzulutskov, A. Breskin, R. Chechik, G. Garty, F. Sauli, and L. Schekhtmann, "The GEM photomultiplier operated with noble gas mixtures," Nucl. Instrum. Meth., vol. A 443, pp. 164-180, 2000.

[17] J. M. Maia, J. F. C. A. Veloso, R. E. Morgado, J. M. F. dos Santos, and C. A. N. Conde, "The micro-hole-and-strip plate gas detector: Experimental results," IEEE Trans. Nucl. Sci., vol. 49, pp. 875-880, 2002.

[18] J. F. C. A. Veloso, J. M. Maia, L. F. Requicha Ferreira, J. M. F. dos Santos, A. Breskin, R. Chechik, and R. de Oliveira, "Recent advances in X-ray detection with micro-hole \& strip plate detector," Nucl. Instrum. Meth., vol. A 524, pp. 124-129, 2004.

[19] J. M. F. dos Santos, J. A. M. Lopes, J. F. C. A. Veloso, P. C. P. S. Simões, T. H. V. T. Dias, F. P. Santos, P. J. B. M. Rachinhas, L. F. Requicha Ferreira, and C. A. N. Conde, "Development of portable gas proportional scintillation counter for X-ray spectrometry," X-Ray Spectrom., vol. 30, pp. 373-381, 2001. 